\title{
LV-PP-3-7
}

\section{Impact of extended living donor criteria focusing on donor safety in living donor liver transplantation}

\author{
Joodong KIM* ${ }^{*}$ Donglak CHOI, Eunkyoung JWA
}

\begin{abstract}
Division of Hepatobiliary Pancreas Surgery and Liver Transplantation, Department of Surgery,
\end{abstract}
Catholic University of Daegu College of Medicine, Daegu, Korea

Introduction: Donor safety has been the primary concern during living donor liver transplantation (LDLT) and therefore most centers keep the strict selection criteria for donor safety. Recently, conventional donor selection criteria have been modified to expand the donor pool in LDLT. Herein, we describe out center's experience for extended living donor criteria focusing on remnant liver volume (RLV) and graft steatosis.

Methods: We retrospectively reviewed the outcomes of 452 living donor right hepatectomy (LDRH) including 115 extended criteria donors who performed at our institution from January 2010 to June 2020. Extended donor was defined with criteria as follows: 1) old donor (age $>40$ years) with RLV of $<35 \%$; 2) young donor (age $\leq 40$ years) with RLV $<29 \%$ and minimal fatty change $(<15 \%) ; 3$ ) young donor with mild hepatosteatosis $(15 \%-30 \%)$ and RLV of $<35 \%$. The outcomes in extended living donors were compared with those in living donors under conventional criteria.

Results: Posthepatectomy liver failure (PHLF) occurred in 48 donors (10.6\%) and most cases were grade A except one case with conventional criteria. PHLF and major complications were not more frequent in extended donor group . In multivariate analysis, the only the event for major complications was associated with PHLF but neither extended criteria nor RLV ratio was related to PHLF.

Conclusions: LDRH under our extended criteria could be performed to expand donor pools without adverse effects on donor safety and could be performed safely in donors with RLV ratio $<30 \%$ under our strict criteria when no other donors are available. 